\section{Genomics and economics}

I t has always been difficult for medical practice to move in lockstep with medical science: the pace of discovery, the rapid translation of new knowledge into innovative technologies and the flood of data that washes over medical practitioners daily are familiar phenomena to us all. Nowadays, these challenges seem particularly acute. It is not only the clinician in the office who struggles to keep up, but the entire health care system.

Nowhere is this more evident than in our understanding of the genetically determined molecular substrates of disease. Traditional pathophysiologic definitions and categories are being segmented into multiple genetic variants that differ markedly in clinical course and treatment response. What used to be simply "breast cancer" is now an expanding set of molecularly definable subcategories. For example, in roughly $25 \%$ of women with breast cancer the human epidermal growth factor receptor gene (HER-2) is amplified; this "HER-2 positive" status increases the risk of metastases and recurrence (see page 347). Trastuzumab (Herceptin), a drug engineered to block cellular HER-2 receptors, has been demonstrated to benefit women with metastatic breast cancer.

Now, as-yet-unpublished results of 2 clinical trials of trastuzumab adjuvant therapy show $50 \%$ reductions in disease recurrence among women with much earlier stages of breast cancer. Trastuzumab therapy has been funded for the last 6 years or so only for patients with advanced metastatic cancer. The recent unpublished results have prompted patients, some leading oncologists and, not insignificantly, the media, to lobby provinces to cover the expanded indications despite the substantial annual costs (\$35000 to $\$ 45000$ per patient). Like dominoes, the provinces are falling in line to authorize provincial funding for the new indications without a completed scrutiny of the evidence base.

So far, patients who have been waiting for access to trastuzumab, agalsidase beta, erlotinib and other therapies have experienced an agonizing mix of hope and frustration. The understandable desire of patients to grasp at what may be their best chance for recovery is now in tension with the need for public insurers to marshall resources in a way that achieves the most cost-effective results for the most people. It is clear that new treatments will put governments in a state of fiscal hopefulness and frustration.

How will we reach a societal consensus about what treatments should be covered by the public purse, and how quickly? Will it be those disease-specific patient groups that are the most successful in raising media awareness, the most adept at cultivating alliances with industry and the most successful at competing for philanthropic donations and attracting researchers who, at the end of the day, set research agendas and drive public funding decisions? Progress in developing new products for rare diseases and for newly recognized risk strata of existing disease would undoubtedly be slower or doomed to permanent neglect without a patient lobby.

Today's patients are savvy and anything but complacent; gone are the days when ivory-tower scientists and granting bodies set research agendas independently of public interest (or of industry investment). It is no surprise that wellinformed patients are asking for specific drugs, genetic testing, antenatal and perinatal screening of their children for multiple conditions and so on, long before their physicians and health policy experts have examined the evidence - or even seen it.

Can we draw a fiscal line in the sand of health care funding? The transforming experience in Oregon's Medicaid program resulted from an attempt to draw such a line. Among the least cost-effective treatments excluded from coverage were expensive therapies for cancer, including bone marrow transplants for childhood leukemia. Public reaction to the story of a single child refused treatment for his leukemia rapidly reversed this policy decision. Costeffectiveness and fiscal constraint do not always go with the human grain.

Funding for the new genomic medicines will have to be found within health care budgets - or by permitting the development of private health insurance, with all its inequities. If we recognize that as a society we can't sell the farm for health care then we must face stark choices between expensive treatments for a few or inexpensive treatments for the many (say, statins for people with hyperlipidemia). And in those cases where government does manage, against the political odds, to say no, it will be interesting to see what alternatives our post-Chaoulli health care system will be permitted to deliver. — CMAJ 